\title{
Observational studies of mass loss from AGB stars
}

\author{
Mikako Matsuura \\ Department of Physics and Astronomy, University College London, Gower Street, London \\ WC1E 6BT, United Kingdom \\ email: mikako@star.ucl.ac.uk
}

\begin{abstract}
It is important to properly describe the mass-loss rate of AGB stars, in order to understand their evolution from the AGB to PN phase. The primary goal of this study is to investigate the influence of metallicity on the mass-loss rate, under well determined luminosities. The luminosity of the star is a crucial parameter for the radiative driven stellar wind. Many efforts have been invested to constrain the AGB mass-loss rate, but most of the previous studies use Galactic objects, which have poorly known distances, thus their luminosities. To overcome this problem, we have studied mass loss from AGB stars in the Galaxies of the Local Group. The distance to the stars have been independently measured, thus AGB stars in these galaxies are ideal for understanding the mass-loss rate. Moreover, these galaxies have a lower metallicity than the Milky Way, providing an ideal target to study the influence of metallicity on the mass-loss rate. We report our analysis of mass loss, using the Spitzer Space Telescope and the Herschel Space Observatory. We will discuss the influence of AGB mass-loss on stellar evolution, and explore AGB and PN contribution to the lifecycle of matter in galaxies.
\end{abstract}

Keywords. Stars: AGB and post-AGB, stars: mass-loss, supernovae: general, galaxies: evolution, Magellanic Clouds, dust, extinction

\section{Introduction}

Spitzer Space Telescope (Werner et al. 2004) has opened up a new frontier of studying asymptotic giant branch (AGB) stars beyond the Milky Way. This includes the photometric surveys of the Magellanic Clouds (MCs; Meixner et al. 2006; Gordon et al. 2011) and spectroscopic studies of selected AGB stars in these galaxies (Zijlstra et al. 2006; Sloan et al. 2006; Buchanan et al. 2006; Lagadec et al. 2007; Kemper et al. 2010), and the dwarf spheroidal galaxies (Matsuura et al. 2007; Sloan et al. 2009). Because distances to AGB stars can be determined independently to AGB stars, we can easily estimate luminosities of AGB stars, which is the largest uncertainty parameter to drive the massloss rate for Galactic objects. Further, these galaxies have lower metallicities than the Milky Way, and they are ideal to study how metallicity influences dust composition and mass-loss rate of AGB stars. The typical metallicities of the Large and Small Magellanic Clouds are half and quarter of the Solar metallicity, respectively (Russell \& Bessell 1989). Here we briefly summarise our resent progress of our studies of AGB stars.

\section{Analysis and results of extra-galactic AGB stars}

We have taken the photometric data of the Magellanic Clouds from Meixner et al. (2006) and Gordon et al. (2011) and these were investigated by these many consortia (Blum et al. 2006, Boyer et al. 2011). We have cross-identified the LMC photometric data with spectroscopically known objects in the LMC, including AGB stars, HII regions, YSOs and post-AGB stars (Matsuura et al. 2009). Recent up-dates by Woods et al. (2011) 


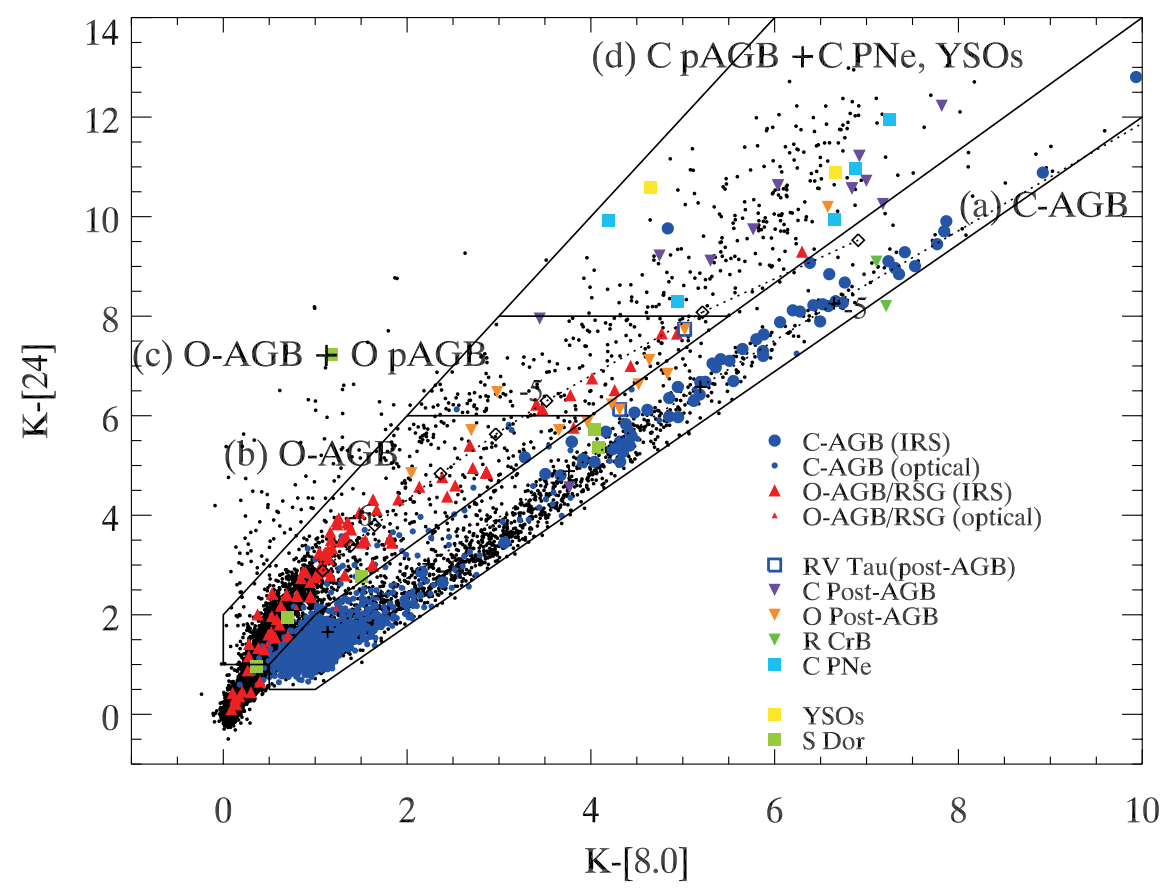

Figure 1. Colour-colour diagramme of point sources in the Large Magellanic Cloud. The objects with spectroscopic identified classifications are overplotted. The sequence of oxygen-rich objects and carbon-rich objects are well separated. Two dotted lines show the estimated mass-loss rate and colour relations for oxygen-rich and carbon-rich stars, respectively.

and Ita et al. (2008) will be incorporated in future work. The resultant colour-colour diagram of the objects in the Large Magellanic Cloud (LMC) is plotted in Figure 1. Sequences of mass-loss rate estimated from Groenewegen et al. (2007), and correlation derived from Groenewegen et al. (2007) are overlaid in dotted lines. The redder colour corresponds to higher mass-loss rate.

Figure 2 shows the same combination of the colour-colour diagram, but for the Small Magellanic Cloud (SMC). There is a slight difference in the distributions of stars, compared with LMC diagram: the SMC has very few oxygen-rich AGB stars with colour redder than $K-[8.0]>1.8$, which corresponds to approximately a mass-loss rate higher than $>10^{-6} M_{\odot} \mathrm{yr}^{-1}$. Near-infrared imaging surveys of the LMC and the SMC, showed that the number ratio of oxygen-rich stars to carbon-rich stars decreases (Richer \& Westerlund 1983), which have similar metallicities, going from the Galaxy (about the Solar), to the LMC (about $1 / 2$ of the Solar metallicity), to the SMC (about 1/4 of the solar metallicity). We found that the impact of the metallicities is not only on the number ratio of oxygen-rich and carbon-rich, but also on the dust formation, and mass-loss rates. After the 3rd-dredge ups, AGB stars might turn from oxygen-rich to carbon-rich, and an circumstellar envelope could have a sufficient number of carbon atoms for the formation of carbonaceous dust grains. Radiative pressure on the dust grains drives the mass loss from AGB stars, thus, mass-loss rates of carbon-rich AGB stars do not reduce significantly towards lower metallicities, at least down to quarter of the solar metallicity. This is consistent with earlier studies based on smaller number of spectroscopic sample (e.g. Matsuura et al. 2005) and theoretical studies (Wachter et al. 2008). However, in oxygen-rich AGB stars, the dominant species of dust grains are silicate, and their mass is mainly limited by the silicon elemental abundance. Silicon atoms are not synthesised 


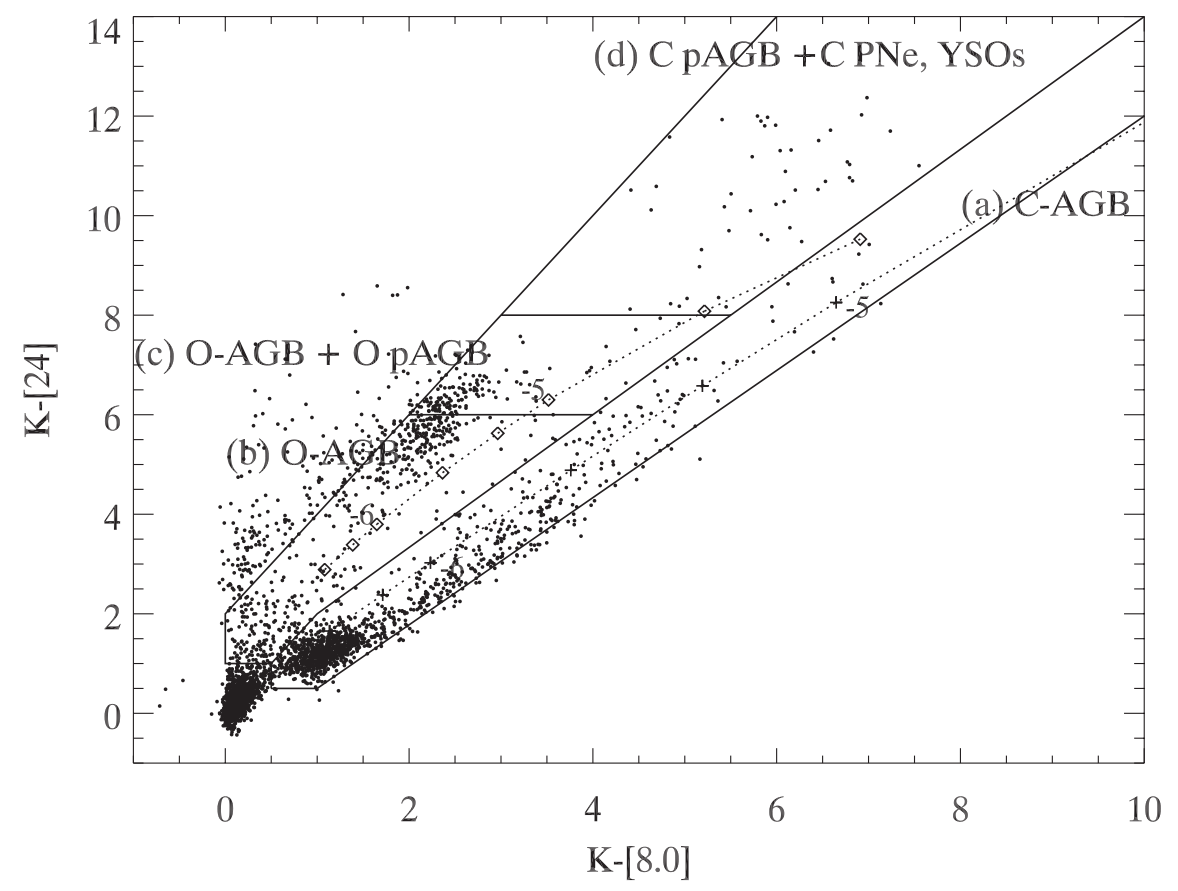

Figure 2. Same as figure 1, but for the objects towards Small Magellanic Cloud (SMC). There are very few number of oxygen-rich stars with colour redder than $K-[8.0]>1.8$, which corresponds to approximately mass-loss rate higher than $>10^{-6} M_{\odot} \mathrm{yr} \mathrm{s}^{-1}$. Note that the cluster of point sources near $\mathrm{K}-[8.0] \sim 2$ and $\mathrm{K}-[24] \sim 6$ are external galaxies located behind the SMC.

inside AGB stars, and their abundance is limited by silicon incorporated into the star when the stars were formed. Silicate abundances in AGB stars largely correlate with the metallicities of the parent galaxies. Thus, the mass-loss rate of oxygen-rich AGB stars reduces towards lower metallicity, as theoretical prediction by Bowen \& Willson (1991) showed. Hence, it is more difficult for oxygen-rich stars to form significant amounts of dust to drive a high mass-loss rate.

Figure 2 shows that the number ratio decrease even drastically towards higher massloss rate stars in the SMC. As the LMC and SMC have experienced more or less similar star-formation histories (Zaritsky \& Harris 2004; Harris \& Zaritsky 2009), the age of stars would not be the main factor to explain this change in number ratio. This is probably caused by the effect of metallicities. It appears that AGB stars change from from oxygenrich to carbon-rich, before they reach a high mass-loss rate, suggesting that the turning from oxygen-rich to carbon-rich might have happened at an earlier age during the AGB stellar evolution. Note that the cluster of point sources near $\mathrm{K}-[8.0] \sim 2$ and $\mathrm{K}-[24] \sim 6$ are external galaxies.

From these mass-loss rates of individual AGB stars, we can estimate the total gas and dust inputs from AGB stars into the interstellar medium (ISM) of the LMC and the SMC. The estimated total gas inputs is $2-4 \times 10^{-2} M_{\odot} \mathrm{yr}^{-1}$ for LMC adding both oxygen-rich and carbon-rich, and $\sim 0.2 \times 10^{-2} M_{\odot} \mathrm{yr}^{-1}$ for the SMC. The estimate particularly for the SMC suffers from contamination of external galaxies, and this requires a future revision and elaborate removal data from these galaxies. The gas inputs from type II supernovae are $2-4 \times 10^{-2} M_{\odot} \mathrm{yr}^{-1}$ for the LMC, and $0.8 \times 10^{-2} M_{\odot} \mathrm{yr}^{-1}$ for the SMC. This estimate is based on the SN rates in these galaxies (Maoz \& Badenes 2010). In the LMC, both AGB stars and SNe contribute comparably to the gas inputs, while in the SMC, AGB 
stars appear to be smaller inputs of gas into the ISM than SNe. Matsuura et al. (2009) found that dust inputs from AGB stars are small, compared with dust present in the ISM of the LMC. Recent finding of dust in SN 1987A (Matsuura et al. 2011) and from the Herschel Magellanic Survey (Meixner et al. 2010) suggests dust inputs from supernovae into the ISM as important contributors.

The mass-loss study of AGB stars continue with Herschel Observations, using the data from the Herschel guaranteed time observations (Groenewegen et al. 2011), which now started revealing a complex structure of the atmosphere (Decin et al. 2010; Royer et al. 2010; Wesson et al. 2010). We are working on modelling spectra using the non-LTE line radiative transfer code, SMMOL (Rawlings et al. 2001).

\section{References}

Blum R. D., Mould J. R., Olsen K. A., et al., 2006, AJ 132, 2034

Boyer, M. L., Srinivasan S., van Loon J. Th., et al., 2011, AJ 142, 103

Bowen G. H. \& Willson L. A., 1991, ApJ 375, L53

Buchanan C. L., Kastner J. H., Forrest W. J., Hrivnak B. J., Sahai R., Egan M., Frank A., Barnbaum C., 2006, AJ 132, 1890

Decin, L., Agúndez, M., Barlow, M. J., et al., 2010, Nature 467, 64

Gordon K. D., Meixner M., Meade M. R., et al., 2011, AJ 142, 102

Groenewegen M. A. T., Wood P. R., Sloan G. C., et al., 2007, MNRAS 376, 313

Groenewegen M. A. T., Waelkens C., Barlow M. J., et al., 2011, A $\& A$ 526, A162

Harris J., Zaritsky D., 2009, AJ 138, 1243

Ita Y., Onaka T., Kato D., et al., 2008, PASJ 60, S435

Kemper F., Woods P. M., Antoniou V., et al., 2010, PASP 122, 683

Lagadec E., Zijlstra A. A., Sloan G. C., et al., 2007, MNRAS 376, 1270

Maoz D., Badenes C. 2010, MNRAS 407, 1314

Matsuura M., Barlow M. J., Zijlstra A. A., et al., 2009, MNRAS396, 918

Matsuura M., Wood P. R., Sloan G. C., et al., 2006, MNRAS 371, 415

Matsuura M., Zijlstra A. A., Bernard-Salas J., et al., 2007, MNRAS, 382, 1889

Matsuura M., Zijlstra A. A., van Loon J. Th., et al., 2005, A $\& A$ 434, 691

Matsuura M., Dwek E., Meixner M., et al., 2011, Science 333, 1258

Meixner M., Gordon K. D., Indebetouw R., et al., 2006, AJ 132, 2268

Meixner M., Galliano F., Hony, S., et al., 2010, A\&A 518, L71

Rawlings J. M. C., Yates J. A., 2001, MNRAS, 326, 1423

Richer H. B. , Westerlund B. E., 1983, ApJ 264, 114

Royer, P., Decin, L., Wesson R., et al., 2010, A\& $\mathcal{A}$ 518, L145

Russell S. C., Bessell M. S. 1989, ApJS 70, 865

Sloan G. C., Kraemer K. E., Matsuura M., et al. 2006, ApJ 645, 1118

Sloan G. C., Matsuura M., Zijlstra A. A., et al., 2009, Sci 323, 353

Wachter A., Winters J. M., Schröder K.-P., Sedlmayr E. 2008, A\& A 486, 497

Werner M. W., Roellig T. L., Low F. J., et al., 2004, ApJS 154, 1

Wesson, R., Cernicharo, J., Barlow, M. J., et al., 2010, A\&A 518, L144

Westerlund B.E., 'The Magellanic Clouds', Cambridge U Press, 1997

Woods P. M., Oliveira J. M., Kemper F., et al., 2011, MNRAS 411, 1597

Zaritsky D., Harris J., 2004, ApJ 604, 167

Zijlstra A. A., Matsuura M., Wood P. R., et al., 2006, MNRAS 370, 1961 